$56^{\text {ème }}$ Congrès de la SFMBCB, 01004 (2011)

DOI: $10.1051 / \mathrm{sfmbcb} / 20115601004$

(C) Owned by the authors, published by EDP Sciences, 2011

\title{
Imagerie et bilan pré - opératoire en implantologie et en chirurgie additionnelle
}

\author{
Felizardo R \\ UFR d'Odontologie Paris 7, APHP Hôtel-Dieu, Paris, France \\ rf95@orange.fr
}

Avec les techniques avancées de chirurgie buccale permettant maintenant de répondre à des situations cliniques jusqu'ici considérées comme relevant de la seule prothèse amovible, de nombreux praticiens étendent leur activité chirurgicale à ce que le Code de Santé Publique nomme les « structures adjacentes » aux arcades dentaires. Cette évolution de la pratique odontologique, parallèlement au renforcement des obligations de moyens qu'elle induit, impose aux praticiens le recours à l'imagerie tridimensionnelle, les examens bidimensionnels conventionnels (rétroalvéolaire, panoramique) ne pouvant assurer une visualisation sans ambiguïté des structures anatomiques, parfois à risque, environnant les sites chirurgicaux. Avec ces techniques avancées pouvant nécessiter plusieurs examens successifs, les praticiens se trouvent confrontés aux obligations de radioprotection et doivent respecter les principes de justification et d'optimisation visant à obtenir le meilleur rapport bénéfice/risque (information/irradiation) pour le patient.

Afin de gérer au mieux cette double obligation, parfois contradictoire, de moyen et de radioprotection, nous discuterons, à partir des données de la littérature internationale et du Guide des Indications et Procédures des Examens Radiologiques en Odontostomatologie publié par 1'HAS en 2006, la stratégie d'exploration à adopter en fonction des situations cliniques et des choix thérapeutiques.

Nous aborderons cette problématique aux différentes étapes du traitement, que ce soit lors de l'exploration du sinus maxillaire avant comblement (volume, cloisons de refend, membrane sinusienne, perméabilité ostiale, présence d'une artère alvéolo-antrale), de la préparation initiale du site implantaire (sites de prélèvement osseux, structures à risque, évaluation du site receveur, faisabilité d'une expansion osseuse), ou de sa réévaluation secondaire (consolidation du greffon, caractéristiques morphologiques finales du site).

Cette problématique sera l'occasion d'aborder la place de la tomographie volumique à faisceau conique (Cone Beam Computed Tomography) dans la pratique chirurgicale, cette technologie qui rend accessible en cabinet privé, sans intervention d'un radiologue, l'imagerie sectionnelle est sans doute destinée à y supplanter progressivement les examens plus irradiants comme le scanner. 